\title{
MuLSi-Co: Multilayer Sinks and Cooperation-Based Data Routing Techniques for Underwater Acoustic Wireless Sensor Networks (UA-WSNs)
}

\author{
Munsif Ali, ${ }^{1}$ Sahar Shah $\mathbb{D},{ }^{2}$ Mahnoor Khan $\mathbb{D}^{3},{ }^{3}$ Ihsan Ali $\mathbb{D},{ }^{4}$ Roobaea Alroobaea $\mathbb{D},{ }^{5}$ \\ Abdullah M. Baqasah, ${ }^{6}$ and Muneer Ahmad $0^{7}$ \\ ${ }^{1}$ Department of Information Technologies, University of Parma, Italy \\ ${ }^{2}$ School of Physics and Electronics, Central South University, Changsha, China \\ ${ }^{3}$ Department of Physics, Government Post Graduate College Nowshera, Pakistan \\ ${ }^{4}$ Department of Computer System and Technology Faculty of Computer Science and Information Technology Universiti Malaya, \\ Kuala Lumpur, Malaysia \\ ${ }^{5}$ Department of Computer Science, College of Computers and Information Technology, Taif University, P. O. Box 11099, \\ Taif 21944, Saudi Arabia \\ ${ }^{6}$ Department of Information Technology, College of Computers and Information Technology, Taif University, P. O. Box 11099, \\ Taif 21944, Saudi Arabia \\ ${ }^{7}$ School of Electrical Engineering and Computer Science (SEECS), National University of Sciences and Technology (NUST), Sector H- \\ 12, Islamabad, Pakistan
}

Correspondence should be addressed to Ihsan Ali; ihsanalichd@siswa.um.edu.my

Received 14 October 2021; Revised 15 December 2021; Accepted 20 December 2021; Published 15 January 2022

Academic Editor: Hamada Esmaiel

Copyright (C) 2022 Munsif Ali et al. This is an open access article distributed under the Creative Commons Attribution License, which permits unrestricted use, distribution, and reproduction in any medium, provided the original work is properly cited.

\begin{abstract}
Designing an efficient, reliable, and stable algorithm for underwater acoustic wireless sensor networks (UA-WSNs) needs immense attention. It is due to their notable and distinctive challenges. To address the difficulties and challenges, the article introduces two algorithms: the multilayer sink (MuLSi) algorithm and its reliable version MuLSi-Co using the cooperation technique. The first algorithm proposes a multilayered network structure instead of a solid single structure and sinks placement at the optimal position, which reduces multiple hops communication. Moreover, the best forwarder selection amongst the nodes based on nodes' closeness to the sink is a good choice. As a result, it makes the network perform better. Unlike the traditional algorithms, the proposed scheme does not need location information about nodes. However, the MuLSi algorithm does not fulfill the requirement of reliable operation due to a single link. Therefore, the MuLSi-Co algorithm utilizes nodes'collaborative behavior for reliable information. In cooperation, the receiver has multiple copies of the same data. Then, it combines these packets for the purpose of correct data reception. The data forwarding by the relay without any latency eliminates the synchronization problem. Moreover, the overhearing of the data gets rid of duplicate transmissions. The proposed schemes are superior in energy cost and reliable exchanging of data and have more alive and less dead nodes.
\end{abstract}

\section{Introduction}

The network structure, reliability, and energy efficiency play an important role in the algorithm designing for UA-WSNs due to its tremendous applications and crucial challenges. A reliable, stable, and efficient algorithm with good network formation is used for many applications, such as military operations, mission, time, and data critical situations, locating and directing submarines and examining oil pipelines and cables $[1,2]$. Moreover, addressing the unique and critical challenges during designing such an algorithm gets stringent attention of the researchers and academia. The acoustic communication amongst nodes tends to extreme latency than the optical and radio communication [3]. But 
optical and radio communication is not feasible for these networks. It is because of the scattering and absorption of these waves [4]. Attaining the location information of the nodes is also challenging due to the constraint of the global position system (GPS) in the water. It is due to the working of GPS on radio waves $[5,6]$. Another way of getting location information is complex simulations. However, this is time and energy consuming, which also introduces computational complexity [7]. Moreover, nodes' movement with water waves degrades the reliable and efficient operation of the algorithm [8]. Furthermore, energy-efficient operations are required due to the constraint of energy. The replacement and energizing of the nodes are hard [9]. The noisy environment in these networks also reduces reliable data exchanging between nodes. These challenges are given in Figure 1. Due to the mentioned reasons, limitations, and challenges, a good algorithm is required for operation of these networks.

Cooperative communication is the best solution in these networks to achieve reliable communication amongst the nodes and reduces data failure. The data advancement through multiple paths towards the destination for increasing the chances of correct data reception is called cooperation [10]. The data advancement every time by the relay for data reliability is called fixed cooperation. While in the incremental cooperation, the delivery is done when the receiver demands from it. Before advancing data to the forwarder/receiver, either it is boosted or decoded by the relay. Based on relay behavior, it is named as amplify forward (AF) and decode forward (DF) [11]. The DF is complex in computation than AF. The cooperative algorithms obtain greater data reception and less packet drop. However, excessive energy and time consumption make these challenging and difficult. In the case of acoustic waves, it is more challenging because of energy resources and the low speed of communication.

In noncooperative algorithms, delivery through a single link consumes less energy and time than cooperative techniques. However, these algorithms are not reliable and have greater chances of packets loss [12]. A small obstacle may lead to data loss and failure. Moreover, noise sources also cause data corruption and have greater effects on data loss in noncooperative algorithms. The retransmission mechanism is not a suitable choice for data reception again over the same noisy link. Also, multiple antennas are costly and not feasible in underwater [13]. Cooperation is the best choice to achieve reliable and good communication in UAWSNs.

The network structure has greater effects on routing performance, especially in UA-WSNs. Poorly designed network leads to bad performance, while a well-designed network structure improves the results and performance. Furthermore, sink placement plays an important role in algorithm efficiency. Sink placement at the best position tends to perform enhancement. Many algorithms such as [14-17] designed network in a single solid structure and placed the sink on the top. Placement of the sink on such positions leads to high and imbalance data load. The high load tends to traffic congestion and data collision. The collision causes

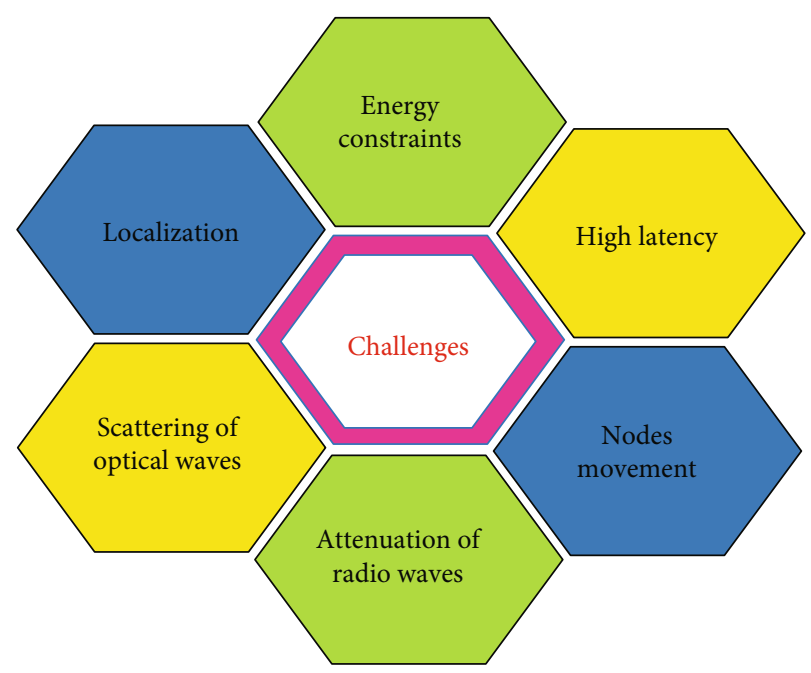

FIgURE 1: Challenges in UA-WSNs.

the packets to drop and forwards data with greater latency. Moreover, the life of the nodes near to the sink is less compared to the other nodes which are far away from the sink.

Many cooperative algorithms exist in the literature [18-24]. Some of the existing cooperative algorithms, such as [25-27], are reliable but they compromise on energy, which leads to less stability and reduces the life of the network. In the case of poorly designed network architecture in cooperative algorithms, its stability is further reduced. Data and time synchronization are other issues in these algorithms. The nodes near to the sink have the highest data traffic. This traffic creates issues, like data collision, a greater amount of energy cost, and high time consumption.

Considering the aforementioned challenges and difficulties, the article proposes two carefully designed algorithms. The first one is the MuLSi algorithm, and the second one is its extended version using the cooperation methodology for reliability enhancement of the MuLSi algorithm. The MuLSi technique proposes a network structure in layers instead of a single solid structure. The sinks are placed in the middle of each layer to reduce the multihop communication between nodes and sinks. Such placement of sinks also reduces the path length between the nodes and the final destination. Reduction in the path length tends to minimize the effects on the data. The next stage consists of data exchanging. The data is delivered to the sink using multihop communication. Therefore, the next forwarder selection is made upon the distance of the nodes from the sink. The node which is the nearest is selected as the next forwarder. In MuLSi algorithm, the data exchanges with the receiver/ forwarder on a single link. The single link does not achieve the best and reliable results. Therefore, cooperation is one of the best solutions to intensify a reliable operation. The article proposes the extended version of the MuLSi algorithm named as MuLSi-Co algorithm. The MuLSi-Co algorithm provides reliable operation by utilizing the broadcast behavior of nodes. The relay forwards data to the destination without any latency. As a result, the forwarder/receiver receives the data along two paths and then combines the 
data to minimize the channel effects and ensure its reliable operation. The overall contributions of the article are summarized as follows:

(i) The network structure plays an important role in network performance improvement. Therefore, the suggested MuLSi algorithm in this article proposes the network structure in layers instead of a single solid structure. Moreover, the layered structure decreases the dependency on other nodes as compared to a single solid structure. Then in each layer, a sink is positioned in the top center. This reduces the multi-hop communication between nodes and tends to reliable and energy-efficient operation. Then instead of the receiver-based approach, the proposed scheme prefers the sender-based approach due to latency, synchronization between nodes, and reduction in computational cost. The sender decides its next forwarder upon nodes' distance from the destination. Instead of complete information about the distance, a simple distance formula is utilized to reduce the computational complexity and cost. Moreover, the knowledge acquisition stage is designed in such a way that reduces the data overhead, traffic congestion, data overload, and energy expenses

(ii) Due to the high effects of the channel on a single link data in the MuLSi algorithm, the chance of data corruption is high. The single link breaking has high chances which affect the reliable transmission and sometimes leads to loss of data. So, this motivation leads to the extended version of the MuLSi algorithm termed as MuLSi-Co. In this, the cooperative behavior of the nodes makes the network reliable. The relay forwards the data to the forwarder/ receiver and combines the copy and original packets to reduce its chances of being dropped

(iii) The redundant packet transmission and synchronization between nodes, sender, receiver, and forwarder, are two of the most important aspects of the cooperative techniques. In order to improve synchronization, a fixed cooperative behavior of the relay tends to eliminate such issues. The relay advances the data as it receives it without any time-wasting. The other nodes in the proximity of the sender discard the data after analyzing it, which leads to a reduction in duplicate data transmission. The suppression of such packets improves efficiency in terms of energy and data collision

\section{Related Work}

The two most important parameters, the power of sensor nodes and less noisy links to transmit data in the harsh environment of the UA-WSNs, are addressed in [28] by proposing two routing protocols. The first scheme "energy effective and reliable delivery (EERD)" deals with the battery consumption parameter, and the second scheme "cooperative energy effective and reliable delivery (CoEERD)" incorporates the noisy link issue. The EERD consists of only one single path, in order to deliver the data from one state to the next higher state. Initially, the source node generates information and advances it. Every source has its own transmission range. All those nodes which come in the range of transmitter receive the data. The source nominates a node (forwarder) amongst the nodes which are around the source for further transmission. The nominating criteria for the forwarder node depends on a weight function. The weight function includes the information of residual energy, bit error rate (BER), and distance. In other words, the node is selected as a forwarder that has maximum residual energy, less BER, and the lowest distance to the sink node. Due to a single link for data transmission, it has the maximum probability of data corruption. To avoid and tackle such issues, an extended version of the EERD is proposed, called CoEERD. In CoEERD, along with the forwarder, a relay is also selected for data advancement to the next stage. The selection criteria of the forwarder and relay nodes are similar as in the EERD mechanism. If the BER increases from the threshold value, then the relay node sends the data. This way improves data exchanging over the noisy links, and the original data is transmitted in a safe mode. The discussed routing schemes outperform, in terms of alive and dead nodes, the successful packet reception with the lowest a lower energy cost.

A "reliable multipath energy-efficient routing protocol (RMEER)" is presented in [29]. This research work targets to enhance the network lifetime and set an optimal route to deliver the information to the desired target. The whole network is divided into five different and equal layers. The final destination node is placed at the top of the water surface, and static powerful carriers are deployed in the remaining layers. The last layer of the network contains ordinary sensor nodes. The multipath data routing mechanism is followed to deliver the information. In order to improve the packet delivery ratio multisinks with the multipath disjoint algorithm are used. In this algorithm, if any node dies, then an alternate route selection bypasses the died node route. The data forwarding process is defined by a routing table. A hello packet is sent by the courier node; after receiving this packet, every source node updates its routing table. This table contains the residual energy, link quality, and node ID. By analyzing all these parameters, an optimal forwarder node is selected for the data transmission towards the sink.

Once the battery of sensor nodes dies, it is impossible to change it in a harsh environment. In addition, the noise degrades the quality of the information packets. These two major problems are incorporated in [30]. For these two problems, two different routing protocols are designed. One deals with a power consumption problem called SRSPR: "stable and reliable short-path routing", and the second deals with the successful transmission of the data called Co-SRSPR. Initially, all the sensor nodes are not familiar with the basic information of the neighbor nodes. So, the hello packet is a method to collect records, all the necessary and basic information of the nodes like residual energy, 
signal to noise ratio, Euclidean distance, and a minimum number of hops. Information in the form of packets is generated and is not sent directly to the sink because of maximum energy consumption and noise. However, it is sent through a multihop mechanism. The best forwarder selection depends on the maximum residual energy, signal to noise ratio, the lowest Euclidean distance to the sink, and minimum hopes. The whole purpose of the mentioned article is to minimize energy consumption. However, this protocol is not able to enhance reliable operation; to do so, a new routing scheme is developed by adding the cooperation to the SRSPR protocol called Co-SRSPR. In Co-SRSPR, one extranode called relay takes part in data transmission in delivery. The relay is selected on the basis of the second largest value of the weight function. If the link is noisy and the BER crosses the limit, then the relay transmits the packet to the final destination. This mechanism minimizes the packet drop ratio and hence increases the successful transmission of the data. Experimental outcomes clearly indicate the best performance of the presented protocol in energy consumption and maximum packet delivery ratio.

In [31], cooperation and multihop energy-efficient routing schemes are introduced for UA-WSNs. The information is generated by the nodes and directs this information towards the sinks through a multihop algorithm. To enhance the reliability of the network, a cooperation scheme is introduced to the one-hop communication. The data forwarding stage is accomplished in two phases. In the first phase, the forwarder node receives the information, and in the second phase, along with the forwarder node, one relay node is set to transmit the data. When both forwarders receive the information, then MRC technique is used which merges these two packets to make one reliable packet. To find the relative distance between nodes, the RSS algorithm is used. The outcomes of the proposed scheme show the best responses in terms of energy and stability of the network.

The fuzzy vector technique is determined in [32] which copes with the delay minimization and battery life issues. This is an advanced version in which fuzzy logic technique (FLT) is utilized. The source generates information and then directs it towards the sink through a multihop mechanism and considers the maximum residual energy for data advancement. The best forwarder selection depends on the residual energy along with the node position. When the data packet generated by the source is broadcasted, all its neighbors receive the packet. Amongst all neighbors, one optimal node is chosen to deliver data to the next node. The residual energy of the selected node should be maximum so that it does not die soon and the position of this node should be minimum to sink node. The experimental results show the best responses in terms of fast data transmission and the network have maximum alive nodes.

In [33], a new mechanism is introduced called "multimedia- and multiband-based adaptation layer techniques" which deals with the bandwidth limitation, propagation delay, and power constraint problems in UA-WSNs. In this routing scheme, the information is collected and transmitted in an effective way. The delivery of data to the sink is accomplished in a hierarchical manner. In order to choose the path, this task is divided into two different phases. The Manhattan approach determines the nodes which are nearer and away from the final destination and selects the medium for data exchanging. The RSSI technique also determines the distance. The modem used for multimedia is developed which supports the bandwidth of acoustic wave range from 70 to $140 \mathrm{KHz}$. Only one single bandwidth is used for infrared wavelength ranges from $700 \mathrm{~nm}$ to $1 \mathrm{~mm}$. For visible light communication, the blue light with 450 to $485 \mathrm{~nm}$ wavelength is used. In the simulation results, the proposed scheme seems better in propagation delay, bandwidth, and energy consumption.

To reduce the interference and undesired signals to the information signal and to use minimum energy by the nodes for transmission and reception of data are proposed in [34]. Multiple datasets are received with minimum or no interference. To control the power parameter of the nodes, a frequency-selective interference channel based on a noncooperative game-theoretic approach is discussed in which the uniqueness and existence of Nash equilibrium are proved. The throughput and cost function of the proposed network is defined by the utility function, in which the cost function indicates the power consumption record of the nodes. The utility function of the nodes changes with the noncooperative mechanism. Hence, increase the throughput and reduce the energy usage of nodes.

Energy-efficient clustering algorithm is proposed in [35], to deal with the balancing and energy problems of the nodes in UWSNs. To reduce the overhead of energy problem during rotation in cluster heads $(\mathrm{CHs})$, leomna, a dual cluster head mechanism, is introduced. To manage the energy of network nodes, a noncooperative game model is also discussed. In this approach, the whole workload in terms of energy is measured first. Then, equal distribution is carried out for each node in terms of energy. In this game model, the Nash equilibrium point is carried out and justified with solid proof. This means a single average value of the energy is defined which every node uses in transmission. Transmission of information amongst intracluster and intercluster domains is carried out in an efficient way to reduce energy usage; to do so, cluster-combined game theory and dual cluster head are proposed, which distribute the energy in an effective way. As a result, the overall network in balancing and energy consumption is optimal compared to existing techniques as shown in the simulation result section.

In [36], network stability and noise awareness are the main concern. This routing called depth-based noise aware scheme targets these two parameters. Initially, the network is idle and knows nothing about nodes. Hello packet is issued from the sink and channel reciprocity obeys in this routing mechanism. By receiving this hello packet, every node puts its ID number, depth, and noise information. By doing this, all nodes become familiar with each other. When the source generates the information signal, every node in the source transmission range will receive this. If all nodes send this data to the final destination node called sink node, then there is huge energy crisis within the network. For this purpose, only one node has been selected to transmit the packet. This node selects the lowest depth and lowest noise 
parameters. Such node is selected up to complete successfully transferring of data to sink node. Hence, the selection of such node reduces the energy consumption, optimizing the path quality of the signal. Moreover, the reliability of the network increases, alive nodes increase, and dead nodes decrease.

Due to the harsh and unpredictable atmosphere of the underwater networks, it hits the overall performance of the network in the aspect of packet delivery, battery life, and the error rate of data. In order to cope with these issues, new ideas have been presented in [37]. This protocol divided the whole network into 4 different regions based on the depth of the nodes. Three different depth-based regions are classified on the base of the depth of the sensor nodes, which are the lowest depth node region, medium depth node region, and highest depth node region. At the top of each region, a sink node is placed which directly communicates with the offshore sink node. Every region has different random nodes amongst which only one node is chosen to transmit the data towards the sink node of each region, respectively. The one forwarder node is selected on the base of the highest residual energy and the lowest depth value. This whole arrangement increases the network time and increases the throughput and reliability of the network.

Due to the most frequent use of top nodes, the energy of those nodes ends which stops further transmission of data; this is called hole generation. The work in [38] avoids the hole generation. Sink nodes are placed far away from the region of interest; therefore, two routing schemes are discussed here, called distributed energy-efficient and connectivity-aware routing protocols. These schemes avoid the mostly used of overhead lowest depth nodes which are responsible to create a hole as a result of which blockage of data transmission in the underwater network takes place. In this protocol, the route changes to multihop. Although the burden of data transmission on the lowest depth nodes minimizes as the output of which the probability of dead ratio in upper surface nodes is reduced, the simulation result shows that the lifespan of the lowest depth nodes increases from 50 to 70 percent. Dead node ratio minimizes, and alive nodes are in maximum number.

In [39], different issues are highlighted and tried to fix them when someone deals with underwater wireless sensor networks. These issues include delay in data processing, more energy usage, and noisy links. However, the most dominant problems are energy consumption and network reliability. An opportunistic energy-efficient routing scheme (EECOR) is proposed here to tackle these problems. In the opportunistic scheme, relay node is selected in cooperative mode with the forwarder node to deliver the data packet in an efficient way. With EECOR, fuzzy logic-based relay is chosen for energy usage and maximum data delivery probability. This scheme avoids the collision of the data packets which reduces the most energy usage of the nodes. The timer mechanism is fixed which notes the time of the already transmitted data packet, and after a fixed time, it sends another packet; in this way, the collision is controlled. The experimental graph shows that the average packet transmission ratio, average delay, and average network lifetime are optimal for the presented protocol compared to the rest schemes.

A multilayer multipath data forwarding scheme is proposed in [40]. Three phases are defined to forwarding the data from bottom to top of the water surface. The first phase explain the network architecture, and in this model, nodes are randomly deployed. In the second phase, the whole routing mechanism is explained, how the data is forwarded to the next higher state nodes. In this phase, the seabed sources initialize the information signal; acoustic powerful nodes are fixed in the upper layer of the surface. The transmitter node is responsible to transmit the data to the acoustic powerful nodes from which the data is directed towards the sink nodes placed on the water surface. The forwarder node selects with lowest depth information; once the forwarder node is selected, this will receive and transmit the information data to powerful acoustic nodes. The RREQ/RREP algorithm is used to choose the best route for multipath transmission. The last phase clearly verifies the shortest distance node selection which sends the data from the source to the sink node. The outcomes show the best performance and maximum improvement in network lifetime, throughput.

DIEER routing is proposed in [41]; it is a delay intolerant energy-efficient algorithm. This protocol can avoid the collision in data packet propagation delay and increase the PDR. No one routing protocol deals with all these problems except the DIEER protocol. The retransmission of data minimizes which reduces the energy usage of the network and hence decreases the delay of the network. The joint optimization framework for sink mobility, hold, and forwarding mechanism is introduced. To reduce the network delay, maximum data delivery, enhancing network life, minimizing energy usage, and the adaptive value of the threshold is fixed with data aggregation and pattern matching schemes. Threedimensional underwater environment is designed with sink mobility and dense deployment of the nodes with varying communication radii. There is no retransmission of the data that occurs by the adaptation of the presented protocol.

In [42], deep learning-coded index modulation-spread spectrum (DL-CIM-SS) technique is adopted to overcome the data rate issue for multiusers and hence remove the flaw that exists in multiuser direct sequence spread spectrum (DSSS). This mechanism sends the data via index of spreading code instead of sending data to all users physically. In this way, the energy usage is reduced, and the maximum data is transmitted. The algorithm proposed in [43] transmits data at a high rate which is the key parameter of underwater communication systems in order to reduce battery power, spreading, and scattering phenomena. The experimental results show the advancement in energy and spectral efficiency.

Secure energy efficiency with cooperation setup is introduced in [18]. The security parameter is not only important for terrestrial networks but also for underwater networks. The security of the data is analyzed for combatting attacks. At the bottom of the network, information has been generated; the main task of the proposed scheme is how to make secure the generated data while transmitting it to the water surface. Along with this, to consume minimum energy in 
transmission and reception, a hello packet is transmitted to all nodes; every node can add the path loss, residual energy, and depth information to this hello packet. The depth threshold from $20 \mathrm{~m}$ to $40 \mathrm{~m}$ is included to avoid the flooding process in the underwater network. Attack of unauthentic signals is checking mechanism to secure the information packet. A duplicate copy of the data packet is already sent to the minisink. When the original data is sent to the sink by multihop process, this original data packet and already sent duplicate copy have been compared. In this way, this protocol ensures the security of the data. This protocol was best performed in terms of security network lifespan. Tables 1 and 2 show the analysis of these algorithms.

\section{The Proposed Scheme}

\subsection{Multilayer Sink Algorithm}

3.1.1. Proposed Network Structure. The network structure has tremendous effects on the performance parameters, such as reliability, stability, scalability, delay, and energy efficiency. The poorly designed network model reduces the overall performance and does not complete the main objectives and aims of the network. So, the network structure plays an important and fundamental role in precise operation to achieve fair and worthy performances. On account of this, a multilayer network structure is proposed as shown in Figure 2. The proposed network is partitioned into five equal layers called a multilayer network. The intention behind the multilayer network structure is to place the sinks on the optimal position, which motive is to accumulate data more efficiently and conveniently. The network comprises of a set $S=S_{n} \cup S_{s}$ of sensor nodes and sinks, where the set of sensor and sink nodes is represented by $S_{n}$ and $S_{s}$, respectively.

The set of nodes $S_{n}=n_{1}, n_{2}, n_{3}, \cdots n_{n}$ is arranged irregularly in the network. These nodes have different sensors for monitoring and investigation of different environmental factors. For transmission of data, each node is equipped with an acoustic modem. The energy at the initial stage of every node is $E_{o}$. Therefore, the total energy of the network is $n$ $\times E_{o}$, where $n$ represents the total number of nodes. Two nodes $x$ and $y$ are neighbors if their transmission range $r_{t}$ is less than its Euclidean distance. All nodes are identical in all aspects, such as energy consumption, data rate, and battery power.

Nodes' random distribution has a negligible effect on performance of the network, while the positioning of the sinks is very important and crucial for performance enhancement, improvement, and data accumulation. So, the sinks $S_{s}=s_{1}, s_{2}, s_{3}, s_{4}, s_{5}$ are placed in the middle top of each layer. The position of the first sink is given as

$$
s_{1}(x, y)=x_{c}, y_{o}
$$

where the $y_{o}$ is the value of $y$ coordinate, and it is 0 for the first sink, while $x_{c}$ represents the center point of the $x$ coor-
TABLE 1: Related work comparison.

\begin{tabular}{lcccc}
\hline Protocol & Energy cost & PDR & Packet drops & Delay \\
\hline Co-EERD & High & High & Low & High \\
RMEER & Low & Low & High & Low \\
Co-RSPR & Low & High & Low & Low \\
CAEEC & Low & High & Low & Low \\
FLVB & Low & Low & High & Low \\
MMMBB & Low & Low & High & Low \\
PCNC & Low & High & Low & Low \\
EECA & Low & High & Low & High \\
EBLFCN & Low & High & Low & Low \\
DEADS & Low & High & Low & Low \\
EEPEH & Low & Low & High & High \\
EECOR & Low & High & Low & Low \\
MLMP & Low & High & High & Low \\
DIEER & Low & High & Low & Low \\
SEECR & Low & High & Low & Low \\
\hline
\end{tabular}

TABLE 2: Related work information.

\begin{tabular}{lcccc}
\hline Protocol & $\begin{array}{c}\text { Location } \\
\text { information }\end{array}$ & Cooperation & $\begin{array}{c}\text { Energy } \\
\text { efficient }\end{array}$ & Multilayers \\
\hline Co- & No & Yes & Yes & No \\
EERD & No & No & Yes & Yes \\
RMEER & No & Yes & Yes & No \\
Co-RSPR & Yes & Yes & Yes & No \\
CAEEC & No & No & Yes & No \\
FLVB & No & No & Yes & Yes \\
MMMBB & No & No & Yes & No \\
PCNC & No & No & Yes & No \\
EECA & Yes & Yes & Yes & No \\
EBLFCN & Yes & Yes & Yes & Yes \\
DEADS & No & No & Yes & No \\
EEPEH & No & Yes & Yes & No \\
EECOR & No & No & Yes & Yes \\
MLMP & No & No & Yes & No \\
DIEER & No & Yes & Yes & No \\
SEECR & & & & \\
\hline
\end{tabular}

dinate in the network and is obtained as

$$
x_{c}=\frac{\text { totallenghtof } x-\text { axis }}{2} .
$$

The positions of the second, third, fourth, and fifth sinks are

$$
\begin{aligned}
& s_{2}(x, y)=x_{c}, y_{o}+a, \\
& s_{3}(x, y)=x_{c}, y_{o}+2 a, \\
& s_{4}(x, y)=x_{c}, y_{o}+3 a,
\end{aligned}
$$




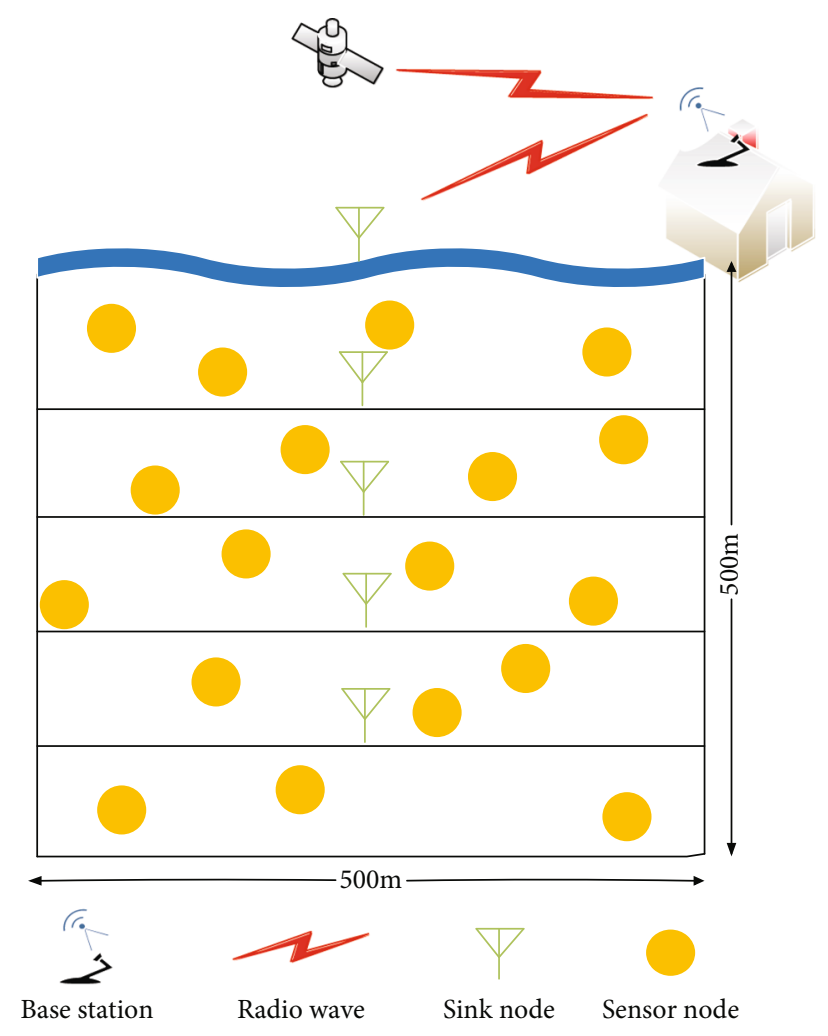

Figure 2: Proposed multilayer network structure.

$$
s_{5}(x, y)=x_{c}, y_{o}+4 a,
$$

where $a=100$. The sink has a greater energy resource than the other nodes. The nodes communicate with each other or sink through acoustic waves with a data rate of $\mathrm{m}$ bit/ sec and frequency $\mathrm{f} \mathrm{KHz}$. The base station collects all the data from the sinks for further processing and analysis as considered in [16].

3.1.2. Knowledge Acquisition. After sinks' and nodes' placement, information about the nodes and sinks are exchanged with each other. In order to minimize the data overhead, traffic congestion, and energy expenditure, information acquisition takes place in parts. The purpose of information or knowledge acquisition is to find the distance of nodes from the sink and their neighbors to select the best candidates for data forwarding. In the first part, the sink broadcasts data of one bit in order to inform the nodes in its transmission area. The node within the transmission area of the sink receives the data. In response to this data, each node transmits INFO PACKET, which contains the ID of that node. As the node broadcasts the INFO PACKET, it set a timer and starts measuring the time. When the sink receives the INFO PACKET, it updates the INFO PACKET by embedding its own information and transmits it towards that node. When the node receives the updated INFO PACKET, it stops the timer and notes its one-way transmission time $t$. The node finds its distance $d$ from the sink by $d=v t$, where $v$ denotes the acoustic wave speed.
In the second part, the other nodes send INFO PACKET and start time counting. In response to this, the nodes which have calculated their distance now respond to other nodes. The node then calculates its distance from each respondent node and its distance from the sink. The sink distance is added to nodes' mutual distance. The INFO PACKET format is given in Figure 3.

In order to enhance network performances, further, updating of information is not done very frequently. However, information acquisition occurred after some interval of time. The updating process is necessary to find its recent and most suitable neighbor for data forwarding.

3.1.3. Data Forwarding. To enhance the network performance, the sender-based approach is a preferred choice in the proposed algorithm. In a sender-based approach, the sender decides its next forwarder which reduces the data forwarding time and computational cost. The reduction of computational cost leads to less energy cost. The nodes near to the sink and lying in sink's transmission area directly communicate with it as shown in Figure 4. These nodes do not need any further route for data delivery. The nodes which are far away from the sink and cannot exchange data with the sink directly follow a multihop communication.

In order to deliver the data quickly, the proposed algorithms follow the shortest route towards the sink by using distance from the destination. Before broadcasting of the data from the sender, it arranges all its neighbours in descending order based on distance information to assign priority to each neighbour. Another word, the nodes having the nearest to the sink, is assigned the first priority for data delivery. When the sender arrange all its neighbours, then it forwards the data packet. Figure 5 depicts and elaborates the packet forwarding scenario. The packet header contains the complete information of the forwarder, final sink, and its own information. The header contains the sender ID, forwarder ID, and the sink ID. After the header data is appended with, they analyze the packet and look for its own ID. When the neighbour ID matches with the ID in the packet, then forward the data packet towards the next stage using the same procedure. The other nodes which receive the data from the sender holds the data for a while, because this data held by the nodes will be utilized in it improved cooperative version of the proposed MuLSi.

The nodes are far away from the sink and follow path to the sink. There are two scenarios of possible position of the neighbours. When these are at different distance from the same, then the sender gives priority to the node which is near to the sink. On another hand, when the nodes are the same distance from the sink, then the sender is free to select anyone of these nodes. Although the second scenario has low chances to occur, it is considered in the proposed algorithm.

It is considered that each sink communicates and forwards its data to the sink placed in the upper layer. The sink placed at the top of the network communicates with the base station. It exchanges all its data with the base station for further processing and analyzing. 


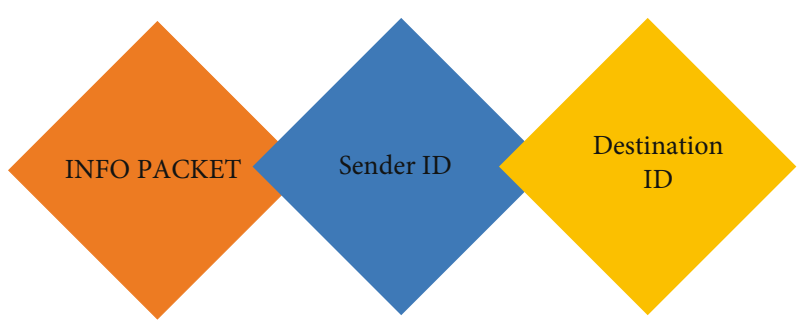

Figure 3: Structure of INFO PACKET.

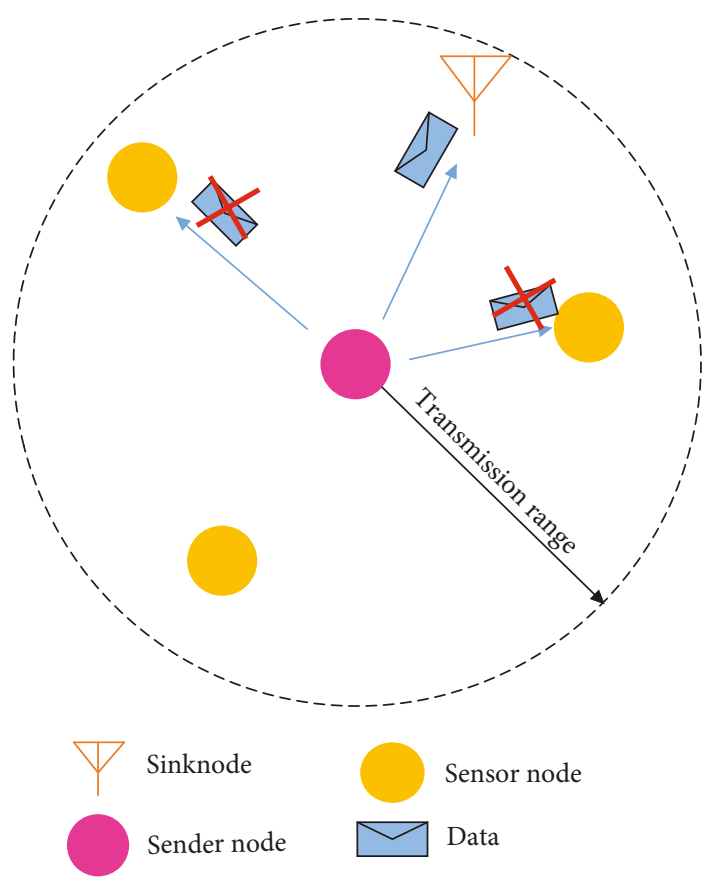

Figure 4: Data exchanging directly with sink.

3.1.4. Suppression of Redundant Packets and Acknowledgment. The proposed algorithms tries to control the redundant packet transmission by utilizing the broadcast nature of the nodes. When these nodes overhear the same packet, then they delete the packet from its memory. They consider that the data is delivered to the next destination. In order to reduce the traffic congestion, data overhead, and energy cost, the acknowledgment is not considered in the proposed algorithm.

3.2. MULSI-Co: Multilayer Sink Algorithm with Cooperation. In the unpredictable and high noise availability in the underwater, a single link is not feasible for communication. The retransmission of the data through the same link is not a good choice, because the data reception through the same link has high chances of data corruption again, which will tend to waste energy and high latency in the data exchanging. The proposed MuLSi algorithm follows a single link for exchanging of data. In order to increase the reliable data reception at the destination of the proposed MuLSi algorithm, the cooperation technique is added to it. Delivery of the same data over many paths towards the destination is known as cooperation. This increases the correct reception

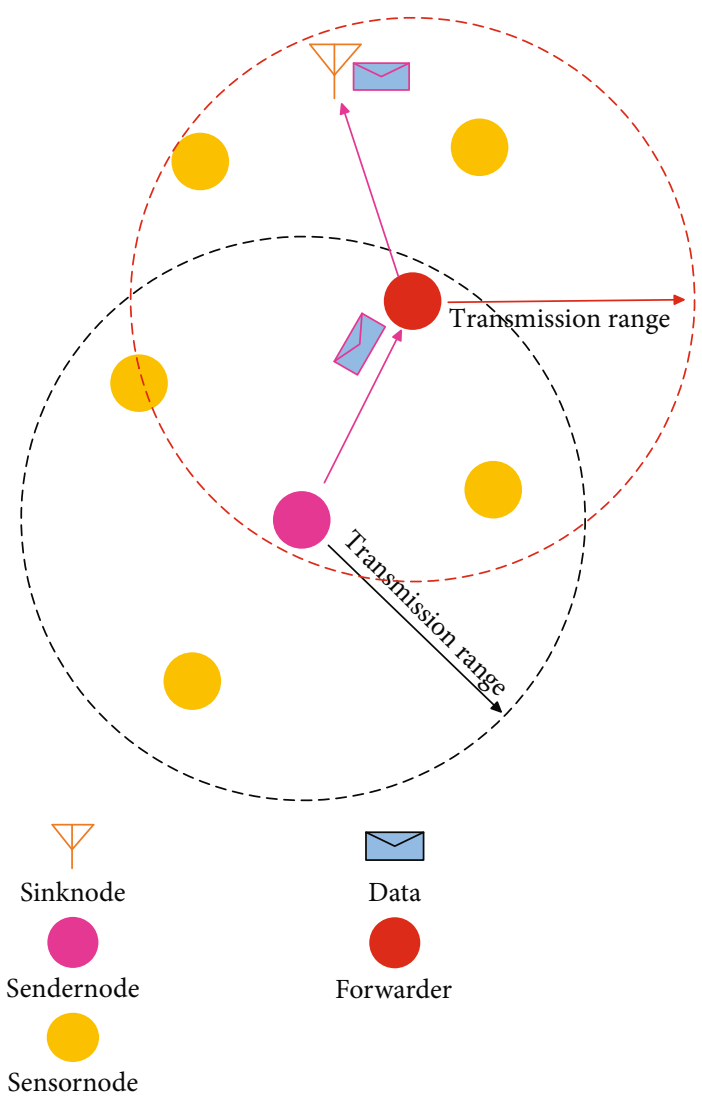

FIgURE 5: Data exchanging with sink through forwarder.

of the data with a cost of more energy and latency than the noncooperative methods. This section gives the details of the proposed MuLSi-Co algorithm.

3.2.1. Cooperative Relay Identification. The important step in cooperation is the relay selection. The best relay selection amongst the neighbors tends to improve the performance. The cooperation technique is reliable, but its cost in terms of energy and delay are greater. So, to minimize the delay and reduce the channel effects, the proposed MuLSi-Co selects the relay which is the nearest to the final destination.

During the knowledge acquisition phase in the MuLSi algorithm, each node has its neighbours list along with their distance information. If the sink is not reachable by the sender directly, then amongst these neighbours, the node which is the nearest to the sink is considered as the forwarder, and the nearer node is selected as relay by the sender. If the sender can exchange its data directly with the sink, then the nearest node is selected as the relay. These relays cooperate with forwarders and destinations to improve reliability.

3.2.2. Data Exchanging and Synchronization. After identification of the relay and forwarder/destination by the sender, the next phase is about data exchanging. Whenever the sender wants to deliver its packet, it appends the information of the relay and forwarder/destination. The sender, relay, and forwarder/destination IDs are appended with the 
data. In case of the direct communication with the sink, the sender appends the ID to it. Otherwise, the node nearest to the sink works as a forwarder, and the sender declares it as a forwarder and appends its ID. And the next node ID is appended as a relay.

Whenever the data is received by any node from any sender, then the node searches for its own ID in the packet. If it finds its own ID in the data as forwarder, this node can work as a forwarder for that sender. The relay also searches for its own ID and works as a relay. When the forwarder receives a packet, it waits for a while to receive its copy from the relay. The data received by the relay is delivered to the forwarder as it is received. The other nodes which hear the data ignore it and become silent. Whenever there is no relay, the forwarder does not wait and deliver it to the next stage without cooperation. The forwarder knows the relay by analyzing the packet.

The time and data synchronization are one of the most challenging stage in cooperation. The delivery of the data by the relay in the exact time is important to improve performance. Therefore, for simplicity, the proposed MuLSi-Co routing is designed in a manner to handle the time synchronization between the relay and forwarder. Whenever the relay receives the data, it forwards it without any wait and holding. Due to the broadcast nature of the nodes, all neighbours hear the data. These neighbors check for its own ID in the data. If they do not find its ID, then they ignore the data. This reduces the duplicate packet transmission and improves the energy cost.

3.2.3. Cooperation and Data Combining Model. In the proposed cooperative algorithm, the data advancement occurs in three steps. In the first step, the sender advances its data to the forwarder and relay. In the second step, the forwarder holds the data for a while and waits for the relay data. As the relay receives data, it sends it to the forwarder. In the third state, two copies of the same data are merged to make one reliable data packet from these.

Considering three nodes, sender $j$, receiver or forwarder $k$, and relay $l$ are depicted in Figure 6 . In the first stage, the $j$ broadcasts the generated data $T_{j}$ towards the $k$ and $l$. The received signal $R j k$ at $k$ is given as

$$
R_{j k}=g_{j k} T_{j}+n_{j k}
$$

The channel noise $n_{j} k$ and the fading $g_{j} k$ affects the data when transmitting from the $j$ to $k$. Likewise, putting a subscript $j_{l}$ to $n$ and $g$ indicates the noise and fading between $j$ and $l$. The received data $R_{j} l$ is represented as

$$
R_{j l}=g_{j l} T_{j}+n_{j l}
$$

In the second stage, the forwarder/receiver $k$ waits for the copy of the same data packet from the $l$. As $l$ receives the data in the first stage, it analyze it. In case the $l$ finds himself eligible for data forwarding, it forwards the data $T_{l}$ to the $k$ without any holding. The factors which influence the data transmission between $l$ and $k$ are the channel noise

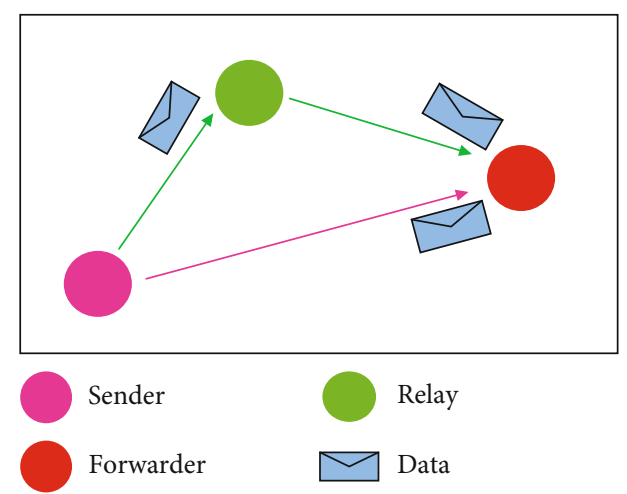

Figure 6: Cooperation model.

TABLE 3: Analysis of the proposed and counterpart algorithms.

\begin{tabular}{lccc}
\hline Protocol & $\begin{array}{c}\text { Routing } \\
\text { parameter }\end{array}$ & Routing & $\begin{array}{c}\text { Network } \\
\text { structure }\end{array}$ \\
\hline DBR & Depth & $\begin{array}{c}\text { Multihop } \\
\text { Multihop }\end{array}$ & Single \\
CoDBR & Depth & $\begin{array}{c}\text { Single } \\
\text { cooperation }\end{array}$ & Multihop \\
MuLSi & Distance & Multi layers \\
MuLSi- & Distance & $\begin{array}{c}\text { Multihop } \\
\text { cooperation }\end{array}$ & Multi layers \\
\hline
\end{tabular}

$n_{l} k$ and fading $g_{l} k$. The $l$ receives the data $R_{l} k$ and is given as

$$
R_{l k}=g_{l k} T_{l}+n_{l k}
$$

In the third stage, $k$ has a direct received packet $R_{j k}$ and its copy $R_{l k}$ received through $l$. Now, the $k$ combines these data using maximal ratio combining. The combined data is given as

$$
R_{k}=R_{j k}+\frac{R_{j l} R_{l k}}{1+R_{j l}+R_{l k}} .
$$

\section{Analysis and Simulation Results}

Validation of the results and network implementation of the proposed schemes are accomplished using MATLAB simulations. The dimension of the network is taken $500 \mathrm{~m}$. The network is further split into five layers having an equal size of $100 \mathrm{~m}^{2}$. The purpose of making such layers is to collect data with easiness and quickly. Moreover, it increases the stability and reliability of these networks. The sink at the top of every layer is placed for data collection and information acquisition of the nodes. The nodes are homogeneous in all aspects scattered randomly in the networks. The total amount of energy on each node is $20 \mathrm{~J}$. These nodes are capable of detecting different environment parameters, for example, temperature and pressures. The LinkQuest UMW 2000 cite modem is coupled with every node for data exchanging. The energy expenditure according to the modem used is considered for transmission and reception are $2 \mathrm{~W}$ and $0.8 \mathrm{~W}$, respectively, while the idle state or sleep 


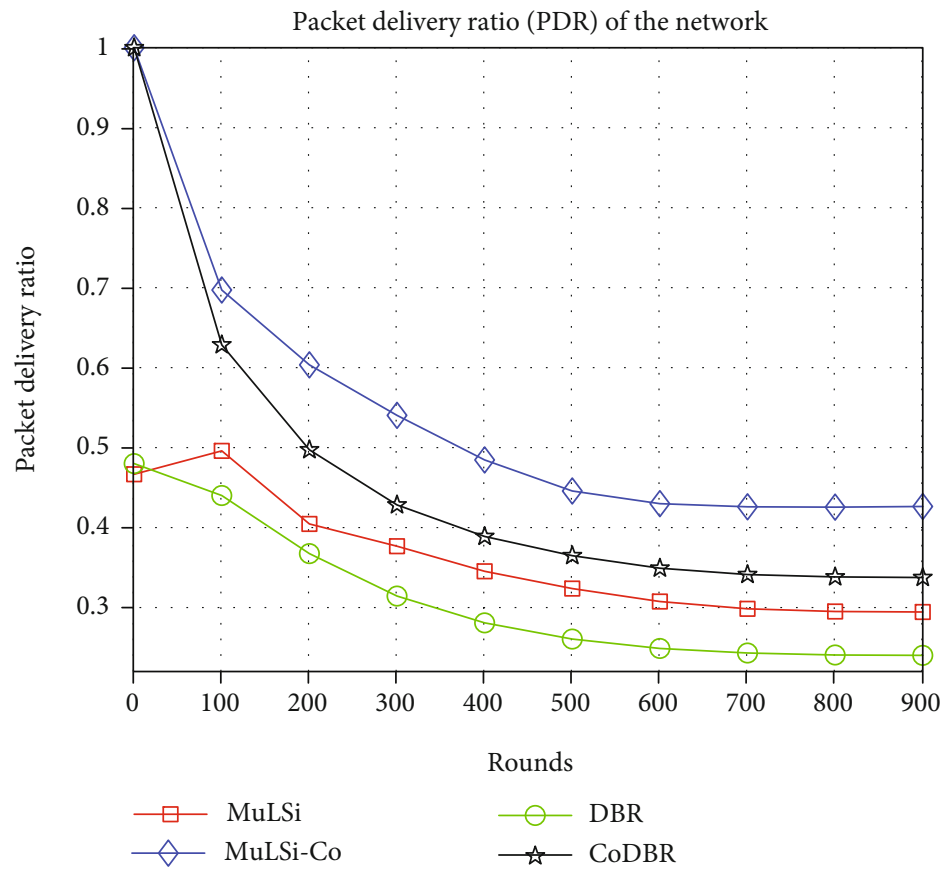

FIgURe 7: Packet delivery ratio (PDR).

TABLE 4: PDR

\begin{tabular}{lcccccccccc}
\hline Protocol & $\begin{array}{c}\text { PDR at } \\
\text { round 1 }\end{array}$ & $\begin{array}{c}\text { PDR at } \\
\text { round 100 }\end{array}$ & $\begin{array}{c}\text { PDR at } \\
\text { round 200 }\end{array}$ & $\begin{array}{c}\text { PDR at } \\
\text { round 300 }\end{array}$ & $\begin{array}{c}\text { PDR at } \\
\text { round 400 }\end{array}$ & $\begin{array}{c}\text { PDR at } \\
\text { round 500 }\end{array}$ & $\begin{array}{c}\text { PDR at } \\
\text { round 600 }\end{array}$ & $\begin{array}{c}\text { PDR at } \\
\text { round 700 }\end{array}$ & $\begin{array}{c}\text { PDR at } \\
\text { round 800 }\end{array}$ & $\begin{array}{c}\text { PDR at } \\
\text { round 900 }\end{array}$ \\
\hline $\begin{array}{l}\text { MuLSi- } \\
\text { Co }\end{array}$ & 1.0000 & 0.6971 & 0.6035 & 0.5403 & 0.4846 & 0.4457 & 0.4299 & 0.4260 & 0.4255 & 0.4264 \\
MuLSi & 0.4667 & 0.4959 & 0.4045 & 0.3767 & 0.3453 & 0.3237 & 0.3075 & 0.2984 & 0.2951 & 0.2944 \\
CoDBR & 1.0000 & 0.6285 & 0.4972 & 0.4283 & 0.3889 & 0.3649 & 0.3492 & 0.3412 & 0.3383 & 0.3375 \\
DBR & 0.4800 & 0.4401 & 0.3674 & 0.3144 & 0.2808 & 0.2606 & 0.2488 & 0.2432 & 0.2407 & 0.2402 \\
\hline
\end{tabular}

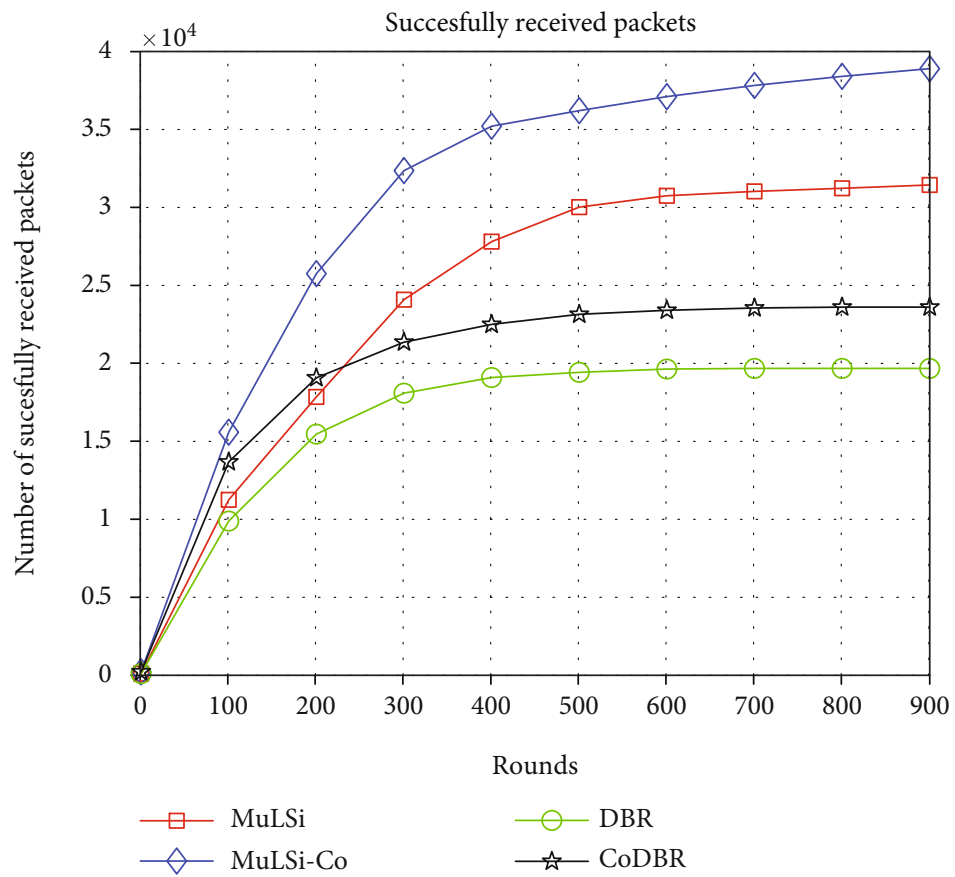

FIgURE 8: Received packets. 
TABLE 5: Received packet analysis.

\begin{tabular}{lcccccccccc}
\hline Protocol & $\begin{array}{c}\text { Received } \\
\text { packets at } \\
\text { round 1 }\end{array}$ & $\begin{array}{c}\text { Received } \\
\text { packets at } \\
\text { round 100 }\end{array}$ & $\begin{array}{c}\text { Received } \\
\text { packets at } \\
\text { round 200 }\end{array}$ & $\begin{array}{c}\text { Received } \\
\text { packets at } \\
\text { round 300 }\end{array}$ & $\begin{array}{c}\text { Received } \\
\text { packets at } \\
\text { round 400 }\end{array}$ & $\begin{array}{c}\text { Received } \\
\text { packets at } \\
\text { round 500 }\end{array}$ & $\begin{array}{c}\text { Received } \\
\text { packets at } \\
\text { round 600 }\end{array}$ & $\begin{array}{c}\text { Received } \\
\text { packets at } \\
\text { round 700 }\end{array}$ & $\begin{array}{c}\text { Received } \\
\text { packets at } \\
\text { round 800 }\end{array}$ & $\begin{array}{c}\text { Received } \\
\text { packets at } \\
\text { round 900 }\end{array}$ \\
\hline $\begin{array}{l}\text { MuLSi- } \\
\text { Co }\end{array}$ & 225 & 15589 & 25755 & 32361 & 35210 & 36208 & 37108 & 37824 & 38406 & 38902 \\
MuLSi & 105 & 11252 & 17850 & 24087 & 27809 & 30021 & 30750 & 31030 & 31229 & 31442 \\
CoDBR & 225 & 13682 & 19076 & 21361 & 22502 & 23148 & 23405 & 23553 & 23610 & 23610 \\
DBR & 108 & 9889 & 15465 & 18092 & 19093 & 19426 & 19638 & 19678 & 19678 & 19678 \\
\hline
\end{tabular}

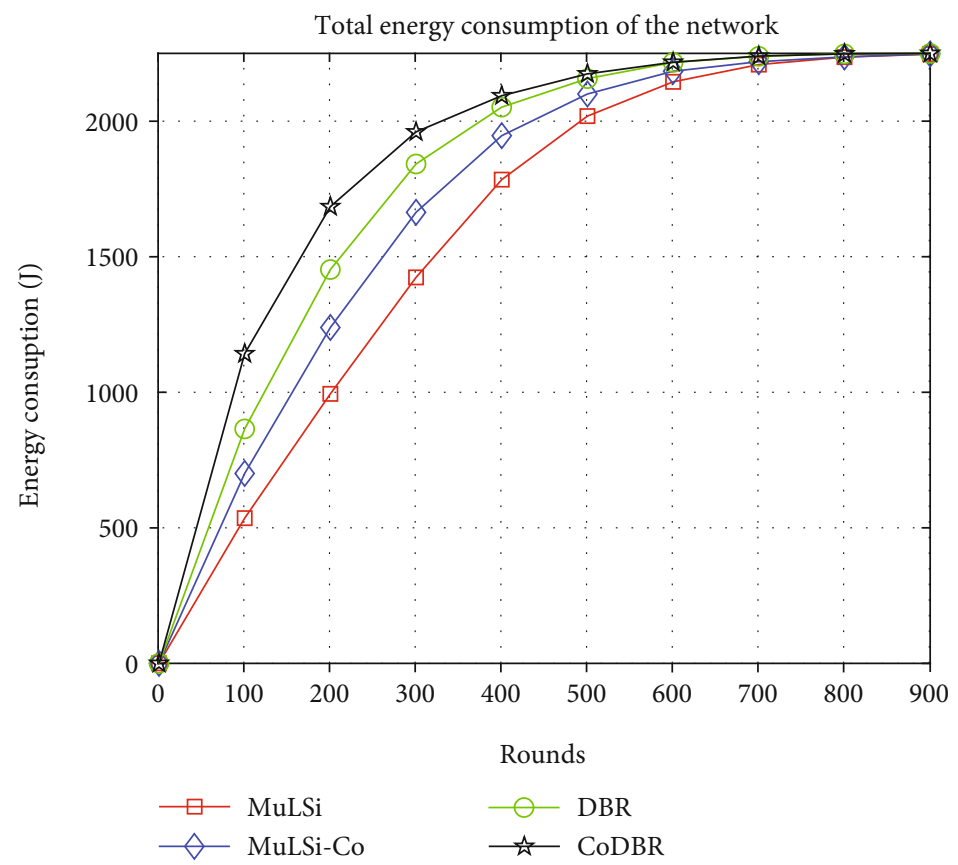

Figure 9: Energy consumption.

TABLE 6: Residual energy analysis.

\begin{tabular}{|c|c|c|c|c|c|c|c|c|c|c|}
\hline Pro & $\begin{array}{l}\text { Residual } \\
\text { energy at } \\
\text { round } 1\end{array}$ & $\begin{array}{l}\text { Residual } \\
\text { energy at } \\
\text { round } 100\end{array}$ & $\begin{array}{l}\text { Residual } \\
\text { energy at } \\
\text { round } 200\end{array}$ & $\begin{array}{c}\text { Residual } \\
\text { energy at } \\
\text { round } 300\end{array}$ & $\begin{array}{c}\text { Residual } \\
\text { energy at } \\
\text { round } 400\end{array}$ & $\begin{array}{l}\text { Residual } \\
\text { energy at } \\
\text { round } 500\end{array}$ & $\begin{array}{c}\text { Residual } \\
\text { energy at } \\
\text { round } 600\end{array}$ & $\begin{array}{c}\text { Residual } \\
\text { energy at } \\
\text { round } 700\end{array}$ & $\begin{array}{c}\text { Residual } \\
\text { energy at } \\
\text { round } 800\end{array}$ & $\begin{array}{c}\text { Residual } \\
\text { energy at } \\
\text { round } 900\end{array}$ \\
\hline Co & $2.2499 \times 10^{3}$ & $1.5577 \times 10^{3}$ & $1.0187 \times 10^{3}$ & 592 & 307.6515 & 151.7722 & 66.7701 & 30.8496 & 13.0675 & 2.4202 \\
\hline MuLSi & $2.2499 \times 10^{3}$ & $1.7196 \times 10^{3}$ & $1.2608 \times 10^{3}$ & 831.2838 & 469.9592 & 234.0915 & 106.1561 & 41.8966 & 14.0033 & 2.8491 \\
\hline CoDBR & $2.2499 \times 10^{3}$ & $1.1205 \times 10^{3}$ & 572.6635 & 293.6711 & 157.3787 & 76.1240 & 33.0757 & 10.1817 & 1.7483 & 0 \\
\hline DBR & $2.2499 \times 10^{3}$ & $1.3937 \times 10^{3}$ & 804.4937 & 412.6768 & 200.1105 & 93.9679 & 33.8725 & 10.9521 & 1.8618 & 0.1186 \\
\hline
\end{tabular}

mode energy exhaustion is $8 \mathrm{~mW}$. Moreover, the data transmission rate is 9600 bps. For the sake of reducing the computational complexity, the data is considered dropped when the channel is busy. The proposed MuLSi and MuLSi-Co schemes are compared with some existing techniques DBR and CoDBR from the literature. The motivation behind choosing these competitive algorithms is the same network architecture, topology, and cooperative behavior of the nodes. An overview of all these algorithms are given in Table 3.

4.1. Packet Delivery Ratio (PDR) or Packet Acceptance Ration (PAR). The ratio of the number of correct packet reception to the total sent packets is termed as PDR or PAR. The 


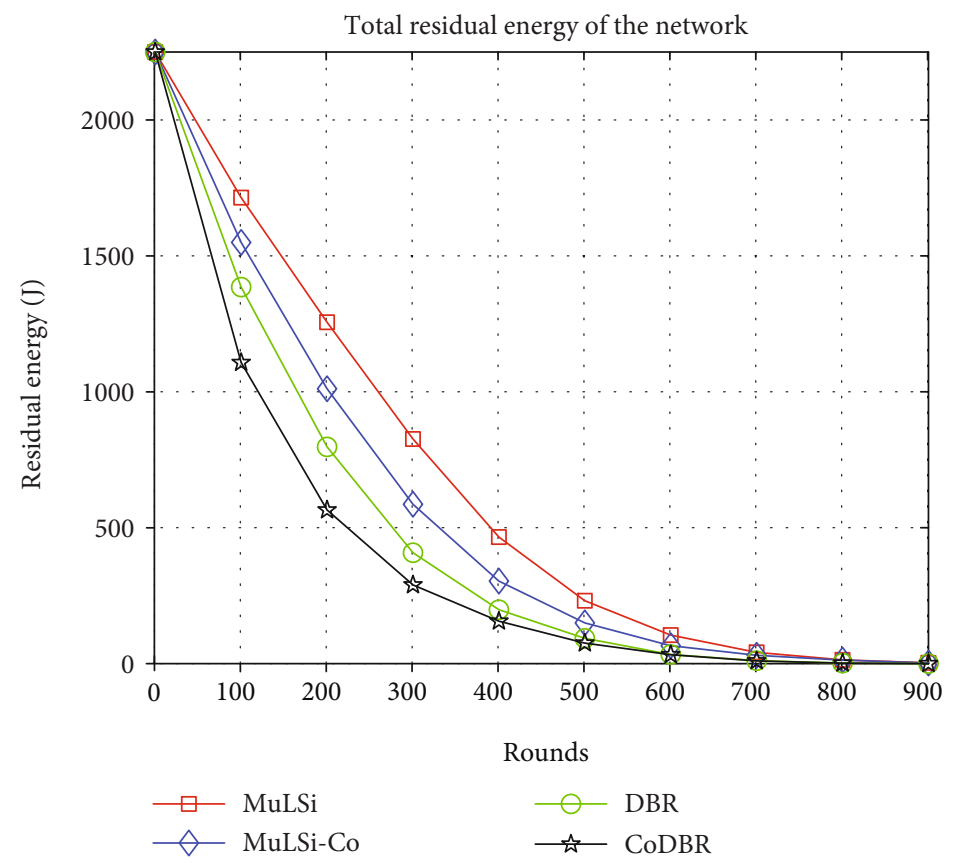

Figure 10: Residual energy.

PDR performance is give in Figure 7. The order of algorithm based on PDR performance from highest to the lowest is MuLSi- Co, CoDBR, MuLSi, and then, DBR. The highest PDR of the proposed MuLSi-Co is due to the sink placement and cooperation. The sink placement in every layer ensures the data reception with the lowest delay than the other schemes, which tends to less effect of the data. Both the MuLSIi-Co and CoDBR are cooperative schemes. However, the sink placement in the proposed cooperative algorithm reduces the path losses and improves the packet reception. In result, the proposed cooperative technique has the highest PDR. Moreover, the best destination and cooperative node selection is another reason of the highest PDR in MuLSiCo algorithm. The second reason behind the highest performance is the cooperation. Incorrect data are exchanged with the retransmitted data by the cooperative node, which makes sure the correct packet reception at the destination.

The CoDBR algorithm PDR has the best performance than the noncooperative schemes, MuLSi and DBR, due to the cooperation and multiple paths are followed for data delivery. The same packets received through many paths are then combined to improve the packet reception and lead to the highest PDR than the noncooperative algorithms.

The MuLSi has the higher PDR than the competitor scheme DBR. It is because of the network topology and node selection using distance parameter for data advancement towards the final destination. The sink placement in each layer and the distance parameter reduce the path for data delivery. This tends to reduce the effects of the channel attributes and increases the probability of correct data reception, while in DBR, the depth is utilized for data forwarding, and the sinks are at the top which are far away from the bottom nodes. Therefore, this increases the channel effect on the data and degrades the packet reception and tends to the lowest PDR. More detail PDR performance is given in Table 4.

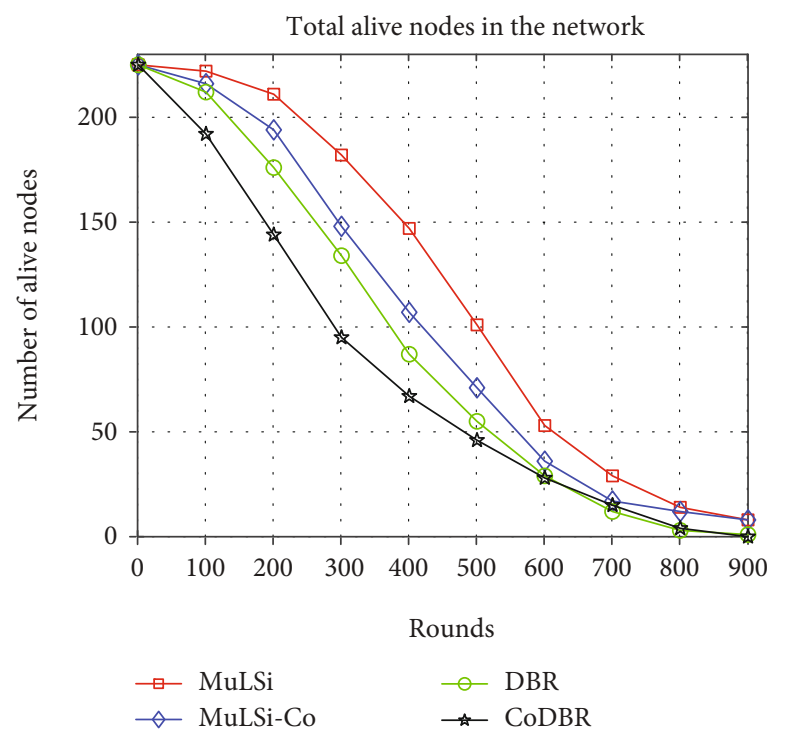

Figure 11: Alive nodes.

4.2. Correct Received Packets. The successful received packet results are shown in Figure 8. The proposed cooperative algorithm has the highest number of received packets. It is due to the data forwarding on multiple paths towards the sink node. The same packet is received through multiple paths and then combined, which increases the probability of correct data reception. Moreover, the sink placement also enhances the probability of correct data reception. Most of the nodes are directly exchange data with the sink. All these parameters lead to the highest data packet reception. 
TABLe 7: Dead node analysis.

\begin{tabular}{lcccccccccc}
\hline Protocol & $\begin{array}{c}\text { Dead } \\
\text { nodes at } \\
\text { round 1 }\end{array}$ & $\begin{array}{c}\text { Dead } \\
\text { nodes at } \\
\text { round 100 }\end{array}$ & $\begin{array}{c}\text { Dead } \\
\text { nodes at } \\
\text { round 200 }\end{array}$ & $\begin{array}{c}\text { Dead } \\
\text { nodes at } \\
\text { round 300 }\end{array}$ & $\begin{array}{c}\text { Dead } \\
\text { nodes at } \\
\text { round 400 }\end{array}$ & $\begin{array}{c}\text { Dead } \\
\text { nodes at } \\
\text { round 500 }\end{array}$ & $\begin{array}{c}\text { Dead } \\
\text { nodes at } \\
\text { round 600 }\end{array}$ & $\begin{array}{c}\text { Dead } \\
\text { nodes at } \\
\text { round } 700\end{array}$ & $\begin{array}{c}\text { Dead } \\
\text { nodes at } \\
\text { round 800 }\end{array}$ & $\begin{array}{c}\text { Dead } \\
\text { nodes at } \\
\text { round 900 }\end{array}$ \\
\hline $\begin{array}{l}\text { MuLSi- } \\
\text { Co }\end{array}$ & 0 & 9 & 31 & 77 & 118 & 154 & 189 & 208 & 213 \\
MuLSi & 0 & 3 & 14 & 43 & 78 & 124 & 172 & 196 & 211 \\
CoDBR & 0 & 33 & 81 & 130 & 158 & 179 & 197 & 210 & 221 \\
DBR & 0 & 13 & 49 & 91 & 138 & 170 & 196 & 213 & 222 & 225 \\
\hline
\end{tabular}

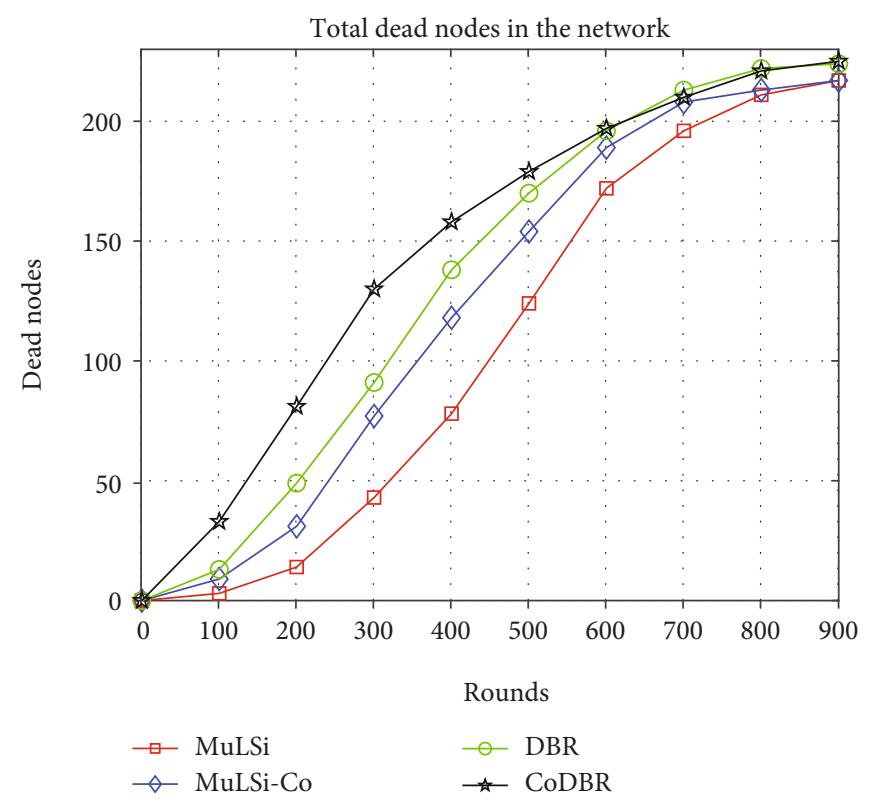

Figure 12: Dead nodes.

The proposed MuLSi algorithm has the highest packet reception than the counterpart algorithms after 230 rounds, although it is a noncooperative algorithm due to well placement of sink nodes and the node selection for data forwarding. The sinks are near to the nodes which exchange data easily, and the channel effects are less on the data. Also, the distance parameter chooses the nearest node to destination. All these factors lead to the highest packet reception.

The CoDBR has the highest packet reception up to 230 rounds. Due to the cooperation of the nodes, the packet reception probability is the highest at the start. Onward, the death ratio of the nodes in CoDBR is high due to the multiple forwards for a single-packet delivery. The multiple forwarders lead to excessive energy consumption and have less chance of cooperation at the end. Therefore, the packet reception becomes lower than the proposed MuLSi algorithm. On the other hand, the DBR has the lowest packet reception due the noncooperative algorithm. The analysis of the packet reception of all these schemes is shown in Table 5 .

4.3. Energy Expenditure and Residual Energy Analysis. The energy expenditure is one of the most important parameter for performance evaluation especially in UA-WSNs. The corresponding results are given in Figure 9. Due to the cooperation mechanism, the consumption of energy in CoDBR is the highest than all other schemes. In cooperation, the data is delivered through multiple paths to the destination. The multipath transmission of the same data tends to consume excessive energy and leads to increase the overall expenditure in terms of energy.

The DBR consumes the highest amount of energy than the remaining algorithms, due the involvement of many nodes for data advancement towards the sink leads to more energy consumption. Another reason of such high-energy expenditure is of the data burden on the top nodes in the network. The top nodes are selected again and again by the lowest nodes to deliver the data. In result, the consumption is the highest than MuLSi-Co and MuLSi. Although MuLSi-Co is a cooperative algorithm, its energy expenditure is lower than the noncooperative algorithm DBR. It is due to the longest route followed for data transmission, and sinks are far away from the bottom nodes in DBR.

The MuLSi-Co has the higher energy consumption than MuLSi algorithm. In MuLSi-Co, for increasing the reliability of the network, the cooperative behaviour of the node is utilised. The cooperation of the nodes tends to consume excessive energy and leads to less network lifetime, while in 
MuLSi, the multilayer network structure and sink placement at the optimal position reduce the path length and node involvement in the data forwarding. Therefore, the resultant energy expenditure of the proposed MuLSi algorithm is the lowest than all other algorithms. The energy performance evaluation is given in detail in Table 6. The above discussion is conversely true for the residual energy and is shown in Figure 10.

4.4. Alive and Dead Node Analysis. The analysis of these algorithms with reference to dead nodes is shown in Figure 11, also with more details in Table 7. The dead nodes in CoDBR algorithm are the highest than the rest of algorithms. As discussed, the network energy expenditure is the highest due to cooperation, and many nodes take part in single-packet delivery. Therefore, in the result, it is concluded that the nodes are spending high amount of energy and tends to greatest number of dead nodes. So, the overall performance of the dead nodes is the highest than the rest of the algorithms.

The DBR has the highest number of dead nodes than the proposed algorithm. For a single-packet delivery, many nodes participate. Due to the involvement of many nodes, they consume a greater amount of energy and tend to increase its death ratio. Therefore, the resultant dead node in $\mathrm{DBR}$ is the highest than the proposed algorithm.

On another hand, both the proposed algorithms have the lowest number of dead nodes than the competitor. Due to the well management of network architecture, sink placement and best node selection during data transmission lead to less energy consumption. The less amount of energy consumption also decreased the number of dead nodes in the network. Therefore, the resultant dead nodes are the highest than the counterpart, while, comparing the proposed noncooperative MuLSi with cooperative MuLSi-Co algorithm, the cooperative algorithm has the higher number of dead nodes. It is because of the greater number of forwarder nodes in the MuLSi-Co algorithm. This discussion is true conversely for the alive nodes and is shown in Figure 12.

\section{Conclusion}

To design and configure an efficient, reliable, and stable algorithm for underwater acoustic wireless sensor networks (UA-WSNs) needs great attention on account of its notable and distinctive challenges and amazing applications. The energy-efficient operation of these networks takes care of their working and running for a longer time and tends to have more stability. The best network structure reduces the number of multiple hops between the sender and destination; this leads to less energy cost and minimized effects of the channel on data. To increase the reliable data exchanging, the cooperation is the best and suitable choice instead of other costly and complex techniques. However, in cooperative techniques, the excessive expenditure of energy makes it challenging. Moreover, the time and data synchronization are also crucial issues in cooperation. To address these difficulties, the article introduces two algorithms: multilayer sink (MuLSi) algorithm and its reliable version MuLSi-Co using the cooperation technique. The first algorithm proposes a multiple-layered network structure instead of a solid single structure and sink placement at the optimal position. This reduces the multiple hops between the sender and sink. Moreover, the best forwarder selection amongst nodes based on the node closeness to sink makes the network performances more better and valuable. The node closest to the sink is a good choice for choosing the best forwarder. However, the MuLSi algorithm does not fulfil the requirement of reliable operation due to a single link for data exchanging. Unlike the traditional algorithms, the proposed scheme does not need the location information about nodes.

\section{Data Availability}

The data used to support the findings of this study are available from the corresponding author upon request.

\section{Conflicts of Interest}

The authors declare that they have no conflicts of interest.

\section{Acknowledgments}

The authors are grateful to the Taif University Researchers Supporting Project (number TURSP-2020/36), Taif University, Taif, Saudi Arabia. This research work was partially supported by the Faculty of Computer Science and Information Technology, University of Malaya, under Postgraduate Research Grant (PG035-2016A).

\section{References}

[1] A. Davis and H. Chang, "Underwater wireless sensor networks," in In 2012 Oceans, pp. 1-5, IEEE, 2012.

[2] R. W. L. Coutinho, A. Boukerche, L. F. M. Vieira, and A. A. F. Loureiro, "Underwater wireless sensor networks," ACM Computing Surveys (CSUR), vol. 51, no. 1, pp. 1-36, 2018.

[3] S. Chafale and N. Dhande, Opportunistic Routing and Monitoring of Packet Data in Underwater Wireless Sensor Network, Tech. Rep, 2018.

[4] J.-H. Cui, J. Kong, M. Gerla, and S. Zhou, "The challenges of building scalable mobile underwater wireless sensor networks for aquatic applications," IEEE Network, vol. 20, no. 3, pp. $12-18,2006$.

[5] A.-K. Othman, "GPS-less localization protocol for underwater acoustic networks," in In 2008 5th IFIP International Conference on Wireless and Optical Communications Networks (WOCN'08), pp. 1-6, IEEE, 2008.

[6] K. Chen, Y. Zhou, and J. He, "A localization scheme for underwater wireless sensor networks," International Journal of Advanced Science and Technology, vol. 4, 2009.

[7] G. Han, J. Jiang, L. Shu, X. Yongjun, and F. Wang, "Localization algorithms of underwater wireless sensor networks: a survey," Sensors, vol. 12, no. 2, pp. 2026-2061, 2012.

[8] M. Ayaz, A. Abdullah, I. Faye, and Y. Batira, "An efficient dynamic addressing based routing protocol for underwater wireless sensor networks," Computer Communications, vol. 35, no. 4, pp. 475-486, 2012. 
[9] A. Wahid, S. Lee, H.-J. Jeong, and D. Kim, "Eedbr: energyefficient depth-based routing protocol for underwater wireless sensor networks," in In International Conference on Advanced Computer Science and Information Technology, pp. 223-234, Springer, Berlin, Heidelberg, 2011.

[10] S. I. Aldharrab, Cooperative Communication Over Underwater Acoustic Channels, 2013.

[11] M. J. Jannati, A. D. Aref, and V. T. Vakily, "Implementation of cooperative virtual MISO communication in underwater acoustic wireless sensor networks," International Journal of Computer Science Issues (IJCSI), vol. 8, no. 4, 2011.

[12] A. Ahmad, S. Ahmed, M. Imran, M. Alam, I. A. Niaz, and N. Javaid, "On energy efficiency in underwater wireless sensor networks with cooperative routing," Annals of Telecommunications, vol. 72, no. 3-4, pp. 173-188, 2017.

[13] L. Lanbo, Z. Shengli, and C. J. Hong, "Prospects and problems of wireless communication for underwater sensor networks," Wireless Communications and Mobile Computing, vol. 8, no. 8, p. 994, 2008.

[14] H. Yan, Z. J. Shi, and J.-H. Cui, "DBR: depth-based routing for underwater sensor networks," in In International conference on research in networking, pp. 72-86, Springer, Berlin, Heidelberg, 2008.

[15] U. Lee, P. Wang, Y. Noh, L. F. M. Vieira, M. Gerla, and J.-H. Cui, Eds., "Pressure routing for underwater sensor networks," in In 2010 Proceedings IEEE INFOCOM, pp. 1-9, IEEE, 2010.

[16] J. Qadar, A. Khan, and H. Mahmood, "DNAR: depth and noise aware routing for underwater wireless sensor networks," in In Conference on Complex, Intelligent, and Software Intensive Systems, pp. 240-251, Springer, Cham, 2018.

[17] Y. Lu, R. He, X. Chen, B. Lin, and Y. Cunqian, "Energy-efficient depth-based opportunistic routing with Q-learning for underwater wireless sensor networks," Sensors, vol. 20, no. 4, p. 1025, 2020.

[18] K. Saeed, W. Khalil, S. Ahmed, I. Ahmad, and M. N. K. Khattak, "SEECR: secure energy efficient and cooperative routing protocol for underwater wireless sensor networks," IEEE Access, vol. 8, pp. 107419-107433, 2020.

[19] Y. Chen, J. Zhu, L. Wan, S. Huang, X. Zhang, and X. Xiaomei, "ACOA-AFSA fusion dynamic coded cooperation routing for different scale multi-hop underwater acoustic sensor networks," IEEE Access, vol. 8, pp. 186773-186788, 2020.

[20] Y. Su, Y. Zhang, R. Bai, Y. Liu, B. Wang, and Y. Sun, Eds., "Qlearning based dynamic cooperative communication in time varying underwater acoustic channels," in In 2021 IEEE/CIC International Conference on Communications in China (ICCC Workshops), pp. 293-298, IEEE, 2021.

[21] S. Karim, F. K. Shaikh, B. S. Chowdhry et al., "GCORP: geographic and cooperative opportunistic routing protocol for underwater sensor networks," IEEE Access, vol. 9, pp. 2765027667, 2021.

[22] H. Zhao, X. Li, S. Han, L. Yan, and Y. Junzhi, “Adaptive relay selection strategy in underwater acoustic cooperative networks: a hierarchical adversarial bandit learning approach," IEEE Transactions on Mobile Computing, p. 1, 2021.

[23] Y. Chen, K. Zheng, X. Fang, L. Wan, and X. Xiaomei, "QMCR: a Q-learning-based multi-hop cooperative routing protocol for underwater acoustic sensor networks," China Communications, vol. 18, no. 8, pp. 224-236, 2021.

[24] R. Arul, R. Alroobaea, S. Mechti et al., "Intelligent data analytics in energy optimization for the internet of underwater things," Soft Computing, vol. 25, no. 18, pp. 12507-12519, 2021.
[25] J. Qadir, U. Ullah, B. Sainz-De-Abajo, B. G. Zapirain, G. Marques, and I. de la Torre Diez, "Energy-aware and reliability-based localization-free cooperative acoustic wireless sensor networks," IEEE Access, vol. 8, pp. 121366-121384, 2020.

[26] A. Khan, I. Ali, A. U. Rahman, M. Imran, and H. Mahmood, "Co-EEORS: cooperative energy efficient optimal relay selection protocol for underwater wireless sensor networks," IEEE Access, vol. 6, pp. 28777-28789, 2018.

[27] H. Nasir, N. Javaid, S. Hifsa Ashraf, Z. A. Manzoor, U. Q. Khan, and M. Sher, "CoDBR: cooperative depth based routing for underwater wireless sensor networks," in In 2014 Ninth International Conference on Broadband and Wireless Computing, Communication and Applications, pp. 52-57, IEEE, 2014.

[28] U. Ullah, A. Khan, M. Zareei, I. Ali, H. A. Khattak, and I. U. Din, "Energy-effective cooperative and reliable delivery routing protocols for underwater wireless sensor networks," Energies, vol. 12, no. 13, p. 2630, 2019.

[29] M. Ahmed, M. Mazleena Salleh, I. Channa, and M. F. Rohani, "RMEER: reliable multi-path energy efficient routing protocol for underwater wireless sensor network," International Journal of Electrical Computer Engineering, vol. 8, no. 6, p. 4366, 2018.

[30] U. Ullah, A. R. Shahid, M. Irfan, J. Qadir, M. Nawaz, and R. Qureshi, "A stable and reliable short-path routing scheme for efficient acoustic wireless sensor networks (AWSNs)," IEEE Access, vol. 8, pp. 1458-1474, 2020.

[31] H. Tran-Dang and D.-S. Kim, "Channel-aware energyefficient two-hop cooperative routing protocol for underwater acoustic sensor networks," IEEE Access, vol. 7, pp. 6318163194, 2019.

[32] R. Bu, S. Wang, and H. Wang, "Fuzzy logic vector based forwarding routing protocol for underwater acoustic sensor networks," Transactions on Emerging Telecommunications Technologies, vol. 29, no. 3, article e3252, 2018.

[33] D. R. KM, S.-H. Yum, E. Ko, S.-Y. Shin, J.-I. Namgung, and S.H. Park, "Multi-media and multi-band based adaptation layer techniques for underwater sensor networks," Applied Sciences, vol. 9, no. 15, p. 3187, 2019.

[34] R. Sun, Z. Wei, X. Zengwei Lyu, L. S. Ding, and H. Songhua, "Power control algorithm based on non-cooperative game theory in successive interference cancellation," Wireless Networks, vol. 25, no. 6, pp. 3297-3305, 2019.

[35] D. Lin and Q. Wang, "An energy-efficient clustering algorithm combined game theory and dual-cluster-head mechanism for WSNs," IEEE Access, vol. 7, pp. 49894-49905, 2019.

[36] J. Qadir, A. Khan, M. Zareei, and C. Vargas-Rosales, "Energy balanced localization-free cooperative noise-aware routing protocols for underwater wireless sensor networks," Energies, vol. 12 , no. 22 , p. $4263,2019$.

[37] A. Umar, N. Javaid, A. Ahmad et al., "DEADS: depth and energy aware dominating set based algorithm for cooperative routing along with sink mobility in underwater WSNs," Sensors, vol. 15, no. 6, pp. 14458-14486, 2015.

[38] R. E. Mohemed, A. I. Saleh, M. Abdelrazzak, and A. S. Samra, "Energy-efficient routing protocols for solving energy hole problem in wireless sensor networks," Computer Networks, vol. 114, pp. 51-66, 2017.

[39] R.,. M. Arifur, Y. Lee, and I. Koo, "EECOR: an energy-efficient cooperative opportunistic routing protocol for underwater acoustic sensor networks," Access, vol. 5, pp. 14119-14132, 2017. 
[40] M. Ahmed, N. Naeem, S. Parveen, N. Hussain, and R. Malookani, "M2DFR: multi-layer multipath data forwarding routing protocol for underwater wireless sensor network," Indian Journal of Science and Technology, vol. 12, no. 1, pp. 16, 2019.

[41] K. Latif, N. Javaid, I. Ullah, Z. Kaleem, Z. Abbas, and L. D. Nguyen, "DIEER: delay-intolerant energy-efficient routing with sink mobility in underwater wireless sensor networks," Sensors, vol. 20, no. 12, p. 3467, 2020.

[42] Z. A. H. Qasem and H. A. Leftah, "Deep learning-based code indexed modulation for autonomous underwater vehicles systems," Communications, vol. 28, article 100314, 2021.

[43] Z. A. H. Qasem, H. Esmaiel, H. Sun, J. Wang, Y. Miao, and S. Anwar, "Enhanced fully generalized spatial modulation for the internet of underwater things," Sensors, vol. 19, no. 7, p. 1519, 2019. 